\title{
Artificial urinary sphincter erosion: the role of corticosteroids in an unusual presentation
}

\author{
Stanley A. Yap, MD; Anthony R. Stone, MD
}

\begin{abstract}
Glucocorticosteroid use has proven beneficial for the management of many medical conditions. Unfortunately its anti-inflammatory properties also profoundly affect many aspects of wound healing. We present a case of an unusual presentation of an artificial urinary sphincter erosion in a patient treated with chronic high-dose steroids.
\end{abstract}

Can Urol Assoc J 2010;4(5):E144-145

\section{Introduction}

The artificial urinary sphincter (AUS) is the established standard for management of post-prostatectomy incontinence and has improved the quality of life for countless patients. ${ }^{1}$ Its acceptance as the standard is attributable to its high success rates and few overall complications., ${ }^{2,3}$ Urethral erosion is a well-recognized complication typically presenting acutely with infection, pain and urinary leakage. ${ }^{4}$ The use of steroids, though clearly beneficial for many diseases, wreaks havoc on postoperative recovery given its anti-inflammatory properties and profound effects on wound healing. ${ }^{5}$ We present an atypical, insidious onset of urethral erosion from an AUS in a patient on chronic high-dose steroids. This case represents an unusual presentation of a well-recognized complication and is particularly relevant given the prevalence of steroid use in our patients.

\section{Case report}

A 69-year-old male initially presented to our clinic with total urinary incontinence following radical retropubic prostatectomy for carcinoma of the prostate. He was otherwise healthy and active, and after standard evaluation including cystourethroscopy and urodynamic studies, an AUS was successfully placed 18 months after his prostatectomy. The placement of this device resulted in complete continence and a highly satisfied patient.

Unfortunately, this patient was subsequently diagnosed with carcinoma of the thymus presenting as acute myas- thenia gravis (MG) 12 months after the AUS placement. An unexpected hospital course was complicated by prolonged intubation and respiratory support, as well as multiple urethral catheterizations without urethral cuff deflation. After successful thymectomy and medical management with highdose steroids, the patient has undergone a full recovery and his MG has remained in remission.

Once recovered from these medical events, he returned to the urology clinic with new complaints regarding his AUS. He required increasing pumping to empty his cuff. Even more troublesome was the recurrence of small amounts of urinary leakage in certain seated positions, though most of his continence was preserved. The patient had no fever and interrogation of the device demonstrated an appropriately cycling AUS. Despite this, urodynamic evaluation was performed with no evidence of leakage or other abnormality. Given this relatively benign presentation, we had a low suspicion for erosion and elected not to perform cystourethroscopy at this time.

The patient returned 4 months later with worsening complaints. He was experiencing more frequent episodes of positional incontinence and developed perineal swelling that increased over the past 2 months. On examination, there was an obvious cystic swelling of the perineum and also in his scrotum adjacent to the pump. It was non-tender and did not have evidence of infection. He continued to deny fever or other systemic complaints. Evaluation at this time involved a computed tomography scan of the pelvis (Fig. 1) demonstrating multiple cystic structures within the pelvis and scrotum. Perineal fluid aspiration and analysis were consistent with a sterile urinoma. Cystourethroscopy was performed demonstrating a distinct urethral erosion at the 1 o'clock position.

The patient was managed with antibiotics and explant of the device using previous incisions in his perineum and suprapubic region. Fluid collections were evacuated and the wounds were closed loosely around the penrose drains. The patient was left with a urethral catheter and no suprapubic drainage initially. An endoscopic-guided suprapubic tube was placed 8 days later after the patient developed persistant urinary drainage from his perineal incision. The perineal 

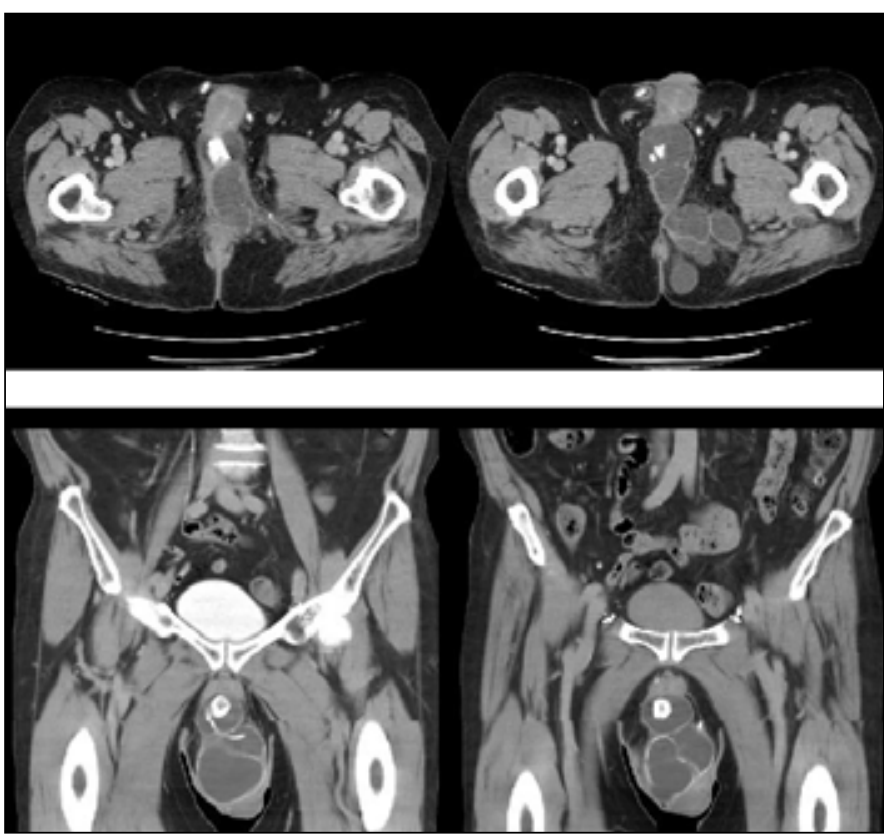

Fig. 1. Axial and coronal computed tomography images of the pelvis demonstrating loculated perineal and scrotal fluid collections, as well as the previously placed artificial urinary sphincter.

drainage subsequently resolved and his suprabupic tube was removed without complication. His Foley catheter was then removed 3 weeks postoperatively.

From the time of his initial hospitalization for MG, the patient was maintained on continuous high-dose steroids with a current dose of prednisone $17 \mathrm{mg}$ per day. His MG has remained in remission. Given his already complicated presentation and concerns for further difficulties with wound healing, his steroid treatment was tapered with the assistance of his neurologist. He was transitioned to primary treatment with mycophenolate mofetil and we initiated a rapid prednisone taper, altering him to every other day dosing and a planned dose reduction of $1 \mathrm{mg}$ every 6 days. He continued his pyridostigmine bromide at a reduced dose. There was no evidence of recurrence of his MG with this medication change.

Despite doing well for a short period after the removal of his catheters, he represented with complaints of a small amount of persistent perineal leakage. Retrograde urethrogram at this time demonstrated a diverticular outpouching at the bulbar urethra. He was taken for operative management where the diverticular capsule was excised and the urethral defect closed with multiple layers. He was left with suprapubic and urethral drainage. Another AUS was successfully placed 4 months after this final procedure.

\section{Discussion}

Potential complications associated with AUSs are welldocumented in the literature..$^{2,3}$ The presentation of such complications, especially urethral erosion, is not subtle and often characterized by acute infection and leakage. Given this typical profile, our ability to rapidly recognize and manage these complications is often taken for granted.

Multiple confounding factors may affect the degree and nature of these complications, and we are still delineating their relevance. Recent studies have begun to elucidate the importance of these factors including the role of prior radiation, ${ }^{6}$ previous surgery or medical comorbidities. ${ }^{7}$ The role of glucocorticosteroids in wound healing and surgical complication rates is also well-documented throughout the literature, 5,8 yet has not been previously described in relation to patients with an AUS.

The inciting factor for this patient's urethral cuff erosion was likely the traumatic catheterizations without cuff deflation. The chronic use of glucocorticosteroids, though, contributed to the progression of his complication and its atypical, insidious onset. This is a well-recognized problem with an unusual presentation leading to a delayed diagnosis and prolonged course of management. This potentially alters our approach to the surveillance and our threshold for concern in AUS patients on chronic corticosteroids given the unique profile of complications and their potentially muted manifestations.

Department of Urology, University of California Davis Medical Center, Sacramento, CA

Competing interests: None declared.

This paper has been peer-reviewed.

\section{References}

1. Litwiller SE, Kim KB, Fone PD, et al. Post-prostatectomy incontinence and the artificial urinary sphincter: a long-term study of patient satisfaction and criteria for success. J Urol 1996; 156:1975-80.

2. Venn SN, Greenwell TJ, Mundy AR. The long-term outcome of artificial urinary sphincters. J Urol 2000; 164:702-6; discussion 706-7.

3. Lai HH, Hsu El, Teh BS, et al. 13 years of experience with artificial urinary sphincter implantation at Baylor College of Medicine. J Urol 2007;177:1021-5.

4. Duncan $\mathrm{HJ}, \mathrm{Mclnerney} P \mathrm{PD}$, Mundy AR. Late erosion. A new complication of artificial urinary sphincters. $\mathrm{Br}$ J Urol 1993;72:597-8.

5. Anstead GM. Steroids, retinoids, and wound healing. Adv Wound Care 1998;11:277-85.

6. Walsh IK, Williams SG, Mahendra V, et al. Artificial urinary sphincter implantation in the irradiated patient: safety, efficacy and satisfaction. BJU Int 2002;89:364-8.

7. Raj GV, Peterson AC, Webster GD. Outcomes following erosions of the artificial urinary sphincter. J Urol 2006;175:2186-90; discussion 2190.

8. Mehrabi A, Fonouni $H$, Wente $M$, et al. Wound complications following kidney and liver transplantation. Clin Transplant. 2006;20(Suppl) 17:97-110.

Correspondence: Dr. Anthony R. Stone, 4860 Y St., Suite 3500, Sacramento, CA 95817; anthony. stone@uddmc.ucdavis.edu 